\title{
Catheter fracture and cardiac migration of a totally implantable venous device
}

\author{
Kapadia Sumit, Parakh Rajiv, Grover Tarun, Yadav Ajay \\ Department of Vascular and Endovascular Surgery, Sir Ganga Ram Hospital, New Delhi, India
}

Correspondence to: Dr. Sumit Kapadia, E-mail: drsumit_k@rediffmail.com

\begin{abstract}
Totally implantable venous devices (TIVD) are increasingly being utilized for venous access for chemotherapy of oncological patients. These devices considerably improve the quality of life of patients requiring long-term chemotherapy. However, despite the great usefulness of TIVDs, their insertion and maintenance is not free of complications. Many early as well as late complications associated with these devices have been reported. We report an unusual, silent, but potentially hazardous complication of catheter fracture and cardiac migration in a 16 year-old girl, in whom the port had been unused for 9 months before presentation. Percutaneous retrieval was unsuccessful as the catheter end was embedded in the myocardium. The catheter was removed via a midline sternotomy without any further complications. We have also reviewed the literature about the possible mechanism of this complication and discussed methods to recognize and avoid it.
\end{abstract}

Key words: Cardiac migration, Catheter, Chemotherapy, Fracture, Totally implantable venous device

\section{Introduction}

Vascular access is an important aspect of management of patients requiring long-term chemotherapy or total parental nutrition. Partially implantable venous catheters, described earlier by Hickman have been used in oncology setups worldwide. ${ }^{[1]}$ The introduction of totally implantable venous devices (TIVD) has revolutionized the care and quality of life of patients with cancer.

The TIVD consists of a silicone catheter whose distal end is positioned in the superior vena cava (SVC), whereas the proximal end is connected to a port reservoir implanted into subcutaneous tissue. This allows for safe and repeated venous access for blood sampling and administration of chemotherapeutic agents. The TIVDs offer advantages over partially implantable systems: low infection rates and unrestricted freedom in patients' physical activities. ${ }^{[2]}$ Deviceassociated complications include infection, catheter obstruction, and deep venous thrombosis. ${ }^{[3]}$ We report an uncommon complication of asymptomatic cardiac migration of the catheter of a TIVD in a 16-year-old girl. This case highlights an infrequent, potentially serious noninfectious complication associated with these widely used devices. Causes of catheter fracture are discussed and recommendations have been laid down for diagnosis and management of this rare problem.

\section{Case Report}

A 16-year-old female had undergone radical nephrectomy for nephroblastoma, 2 years ago. A TIVD (Chemoport, HDC Corporation, CA, USA) was implanted into her right subclavian vein by a percutaneous technique for adjuvant chemotherapy. She subsequently received nine cycles of chemotherapy. She presented to us in December 2004 for removal of the port, which had been unused for the past 9 months. An infraclavicular incision was made at the site of the palpable subcutaneously placed port. During surgery, the port reservoir was dissected, but the catheter was missing from its expected position. A chest radiograph showed the catheter to have embolised into the heart. 


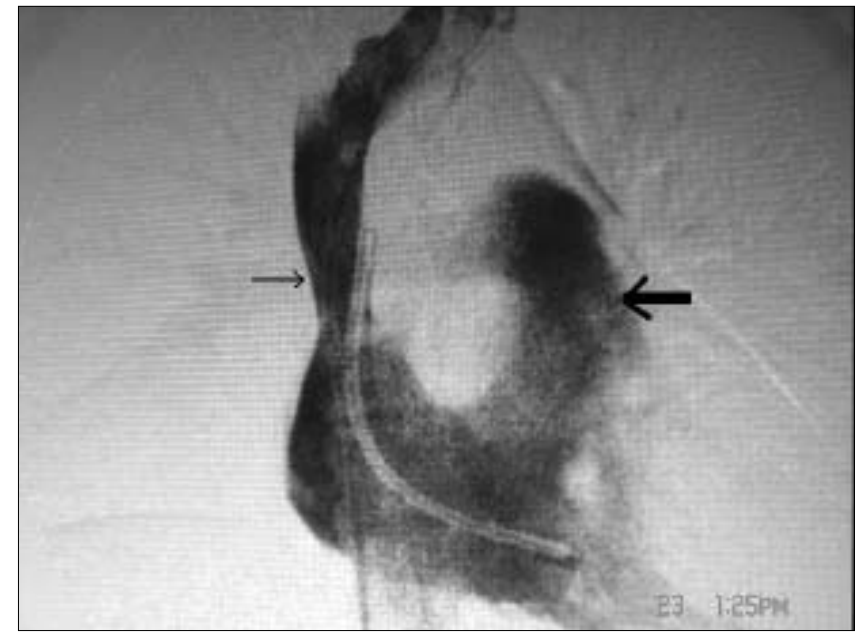

Figure 1: Angiography done via right femoral vein. The catheter is adherent to the wall of SVC (small arrow). The pulmonary artery is also seen (bold arrow)

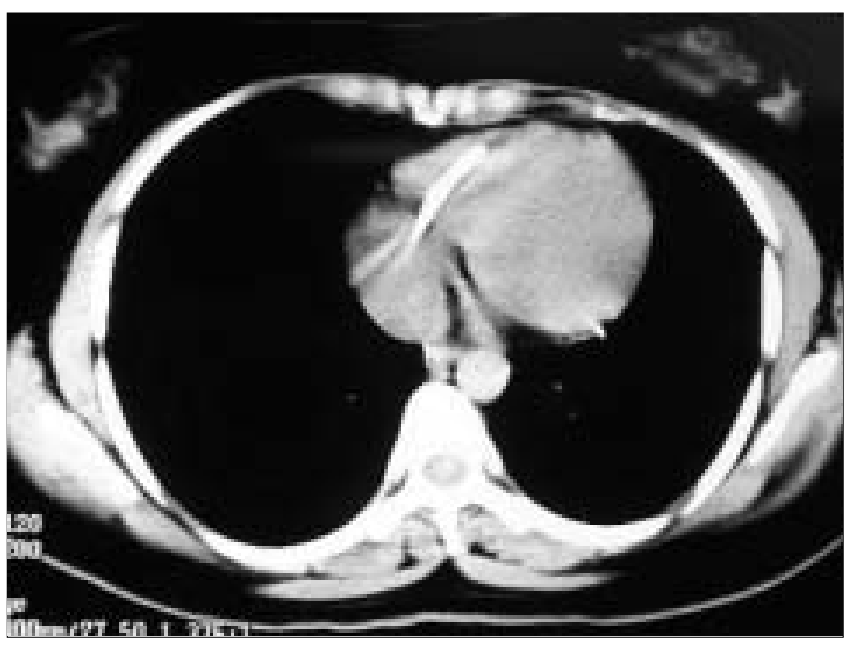

Figure 2: CT Scan shows the catheter embedded in the wall of right ventricle

An attempt to remove the catheter by a vascular snare introduced through the right femoral and right internal jugular veins was unsuccessful. [Figure 1] shows the catheter occupying the SVC (small arrow), right atrium, and right ventricle. A CT scan was obtained to assess the exact relation of the catheter to the cardiac wall [Figure 2]. It demonstrated the distal catheter end to have been embedded into the myocardium of the right ventricle, whereas the proximal end was stuck to the wall of the SVC. The patient subsequently underwent a midline sternotomy with extraction of the catheter through a right atriotomy [Figure 3, arrow]. The postoperative course was uneventful. She is asymptomatic on her 6-monthly follow up.

\section{Discussion}

The TIVD are widely used in patients requiring longterm chemotherapy. Such devices are advantageous in

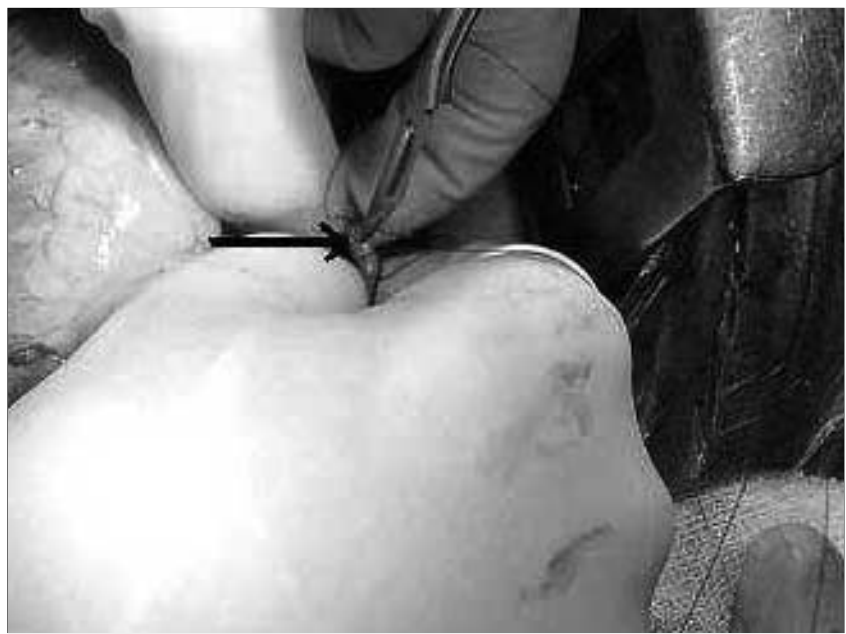

Figure 3: Intraoperative photograph shows the catheter in right atrium (arrow), before atriotomy

that the chemotherapeutic drug is delivered into the central veins via a port, thus avoiding multiple painful venous cannulations and thrombophlebitis. These devices are usually implanted in the cephalic vein or external jugular vein using the cut-down technique, or by percutaneous puncture of the subclavian vein using the Seldinger technique.

Despite the advantages they offer, such devices need expert handling care and may be occasionally associated with complications. Early complications include bleeding, pneumothorax, and wound infection. Catheter infection, catheter obstruction, and deep vein thrombosis are the most important long-term complications. ${ }^{[3]}$ Catheter fracture and dislocation is an uncommon but life-threatening complication with an estimated rate of $0.1 \% .^{[4]}$ Fracture usually follows intermittent compression of catheter between the clavicle and the first rib, especially if the catheter has been inserted too far medially. Further complications of catheter fracture include migration of the catheter fragment and extravascular administration of cytotoxic drugs. The embolised catheter fragment may travel to the right atrium, ventricle, or even into the pulmonary artery. Perforation of caval vein and migration of the catheter tip into lung or mediastinal structures has also been observed. ${ }^{[5]}$

Blood aspiration followed by heparinised saline injection is a standard safety test to be undertaken before administration of chemotherapeutic agents. Local pain or subcutaneous extravasation during port flushing should alert the physician to the possibility of catheter occlusion or fracture. Rarely, it may go unnoticed and present as an intracardiac foreign body with fever or arrythmias. A catheter fragment in the heart may remain asymptomatic for years. ${ }^{[6]}$ 
Catheter fracture may be preceded by the radiographic 'pinch-off sign,' consisting of a compression of the catheter as it passes between the first rib and the clavicle. ${ }^{[7]}$ In a recent collective review of literature, Mirza et al. found 109 cases of this sign, which were detected 1-60 months after insertion. ${ }^{[8]}$ This potentially disastrous complication can be avoided by the early removal of the device if the pinch-off sign is present. ${ }^{[9]}$ A standard chest X-ray can detect catheter dislocation or migration. However, the gold standard for diagnosis is radiographic visualization with contrast injection via the port. This is not a routine procedure before each cycle of chemotherapy as it is associated with the risk of contrast-medium-related complications, need for radiological facilities, and higher costs. Therefore, the most practical recommendation is proper saline testing before use. Any difficulty in this procedure should be evaluated by expert physicians to avoid severe catheterassociated complications. In this patient, the port had not been flushed or used for more than 9 months. Hence, the exact timing of catheter fracture or embolisation could not be estimated. Figure 2 shows one end of the catheter adherent to the SVC, while its other end is embedded in myocardium of the right ventricle.

Percutaneous retrieval methods through a femoral vein approach, using vascular snares are usually suitable and successful for catheter fragments in the right side of heart. ${ }^{[10]}$ Nevertheless, a catheter embedded in the myocardium at either end, like in this patient may need a right anterolateral thoractomy or median sternotomy for removal.

\section{Conclusions}

1. Intracardiac catheter migration is a rare but serious complication of a totally implantable venous access device.

2. These implanted devices require heparinised saline flushing once a month, even when not in use for chemotherapy.

3. Periodic radiographs can be obtained to detect the pinch-off sign.

4. This complication can be avoided by using the cephalic vein cut-down technique.

5. Any implanted catheter should be removed after completion of treatment, or the system's integrity monitored on a regular basis.

\section{References}

1. Hickman RO, Buckner CD, Clift RA, Sanders JE, Stewart P, Thomas ED. A modified right atrial catheter for access to the venous system in marrow transplant recipients. Surg Gynecol Obstet 1979; 148:871-5.

2. Freytes CO, Reid P, Smith KL. Long-term experience with a totally implanted catheter system in cancer patients. J Surg Oncol 1990;45:99-102.

3. Wolosker N, Yazbek G, Nishinari K, Malavolta LC, Munia MA, Langer M, Zerati AE. Totally implantable venous catheters for chemotherapy: experience in 500 patients. Sao Paulo Med J 2004; 122:147-51.

4. Di Carlo I, Cordio S, La Greca G, Privitera G, Russello D, Puleo S, Latteri F. Totally implantable venous access devices implanted surgically: a retrospective study on early and late complications. Arch Surg 2001;136:1050-3.

5. Hackert T, Tjaden C, Kraft A, Sido B, Dienemann H, Buchler MW. Intrapulmonal dislocation of a totally implantable venous access device. World J Surg Oncol 2005;3:19.

6. Van Den Akker-Berman LM, Pinzur S, Aydinalp A, Brezins M, Gellerman M, Elami A, Roguin N. Uneventful 25-year course of an intracardiac intravenous catheter fragment in the right heart. J Interv Cardiol 2002; 15:421-3.

7. Aitken DR, Minton JP. The "Pinch-off sign": warning of impending problems with permanent subclavian catheter. Am J Surg 1984; 148:633-6.

8. Mirza B, Vanek VW, Kupensky DT. Pinch-off syndrome: case report and collective review of the literature. Am Surg 2004;70:63544.

9. di Carlo I, Fisichella P, Russello D, Puleo S, Latteri F. Catheter fracture and cardiac migration: A rare complication of totally implantable venous devices. J Surg Oncol 2000;73:172-3.

10. Actis Dato GM, Arslanian A, Di Marzio P, Filosso PL, Ruffini E. Post traumatic and iatrogenic foreign bodies in the heart: Report of 14 cases and review of the literature. J Thorac Cardiovasc Surg 2003; 126:408-14. 